\title{
EL TEJIDO TEXTIL TARAHUMARA. Hilos, \\ COBIJA Y FAJA
}

ILIANA ROCÍO MARCELEÑO ÁLVAREZ,

SILVIA VERÓNICA ARIZA AMPUDIA ${ }^{1}$

\section{RESUMEN}

$\mathrm{E}$ ste trabajo trata sobre el textil y la importancia del tejido en los pueblos originarios, especialmente sobre el concepto y uso del hilo en la cobija y la faja de los tarahumaras, una de las etnias más representativas del norte de México. Describe, desde la revisión de algunos trabajos de cronistas y exploradores, así como de investigadores contemporáneos, qué significa tejer y cómo los productos tejidos son parte de la vida cotidiana y los rituales de los rarámuri; ${ }^{2}$ son productos que ayudan a conocer parte de su visión sobre el mundo. Además, se presenta una experiencia de campo con artesanas tejedoras de la Sierra Tarahumara, que permite exponer cómo la elaboración de sus tejidos se relaciona tanto con su cultura como con su visión personal como creadoras.

Palabras clave: textiles, tarahumara, rarámuri, norte de México

1 Rocío Marceleño, licenciada en Diseño para la Comunicación Gráfica, estudiante de la Maestría en Estudios y Procesos Creativos en Arte y Diseño. Verónica Ariza, doctora en Diseño, docente investigadora en el Instituto de Arquitectura, Diseño y Arte de la Universidad Autónoma de Ciudad Juárez.

2 Rarámuri es el nombre que los tarahumaras (término utilizado en español) se dan a sí mismos. En este texto nos referimos a los miembros de esta etnia con un nombre u otro de forma indistinta. 


\begin{abstract}
This work presents textiles and the importance of the fabric among native peoples, especially on the concept and use of the thread in the blanket and the fabric belt of the Tarahumaras, one of the most representative ethnic groups of the north of Mexico. It describes, from the review of some works by chroniclers, explorers, as well as contemporary researchers, what knitting means and how woven products are part of everyday life and rituals of the rarámuri; they are products that allow to know part of his vision about the world. This work also presents a field experience with women handcrafters from Sierra Tarahumara, which allows us to explain how the elaboration of their crafts is related to both its culture and their personal vision as creators.
\end{abstract}

Key words: textiles, tarahumara, rarámuri, northern México

\title{
INTRODUCCIÓN
}

Los textiles han sido testigos de los cambios sociales y culturales de los pueblos; en ellos podemos observar la historia y tecnología que los ha acompañado en su proceso de civilización. Desde el comienzo de su existencia el ser humano ha recurrido a la protección del medio ambiente en el que se encuentra; muchos elementos del contexto en que se mueve le auxiliaron en esta tarea. Los primeros grupos humanos cazadores-recolectores utilizaban pieles de los animales que cazaban y fibras de las plantas que encontraban en su camino. Si bien el hombre imitaba a los animales y podía ver en la construcción de un nido o una telaraña una forma particular de entrelazar materiales para poder generar objetos, fue solo cuando se dio la recolección de algunas fibras duras que se crearon los primeros tejidos formalmente. En México, en tiempos antiguos los grupos otomíes y mazahuas, por ejemplo, utilizaban para confeccionar vestidos el henequén, mismo que se obtiene de las pencas del maguey; por otro lado, los restos textiles en el norte del país muestran datos del uso de plantas del desierto, especies 
de palma de hojas gruesas como agaves y yucas, de las que se sacaban las denominadas fibras duras (Lechuga, 1997, p. 14).

El conocimiento de las plantas y fibras da paso a los primeros desarrollos para el tejido. Mediante esta actividad se crean objetos de primer uso, es de decir, objetos cotidianos, como la cestería y las telas. El primer objeto que da muestra del tejido es la cestería, ejemplo de ello es la tejeduría de fibras duras para crear objetos con el fin de almacenar semillas, el principal de los alimentos del hombre nómada. Otro de los objetos es conocido en México como petate, que tenía un importante uso cotidiano y simbólico: "Las esteras (petates) constituían parte del ajuar de toda la familia, pues se nacía sobre una de ellas, la cual serviría, al morir, como mortaja; se empleaban como tapetes para sentarse a comer y para hacer todo tipo de actividades (religiosas, comerciales, públicas)". (Velasco, 1995, p. 129). Otros artefactos servían para transportar mercancías o como utensilios para la limpieza de la casa o el almacenamiento, o tenían un uso ceremonial.

Los tejidos son reflejo de las actividades del hombre en un momento determinado de su desarrollo en sociedad, pero también mostraban la expresión de las culturas: "el tejido era una actividad creadora que daba vida a la sociedad precortesiana” (StresserPéan, 2012, p. 232). La importancia de los tejidos radica en su visión como elementos culturales y su papel en la conservación de la cosmovisión de los pueblos, ya que plasman una espiritualidad que les permite mantener viva su tradición, sus creencias, ritos y cultura; mediante la indumentaria por ejemplo, se diferencian unos de otros, manteniendo su identidad. Los tejidos son metáforas de cómo están construidos la vida y el universo.

Las artes textiles (entre las que se encuentran la fabricación de esteras y redes tanto como la producción y el tejido de hilos) igual que todo lo creado por el hombre, reflejan los mitos de las culturas, "mitos de la salida y la puesta de sol, mitos de los eclipses, mitos de los terremotos, mitos locales que explican los nombres de los lugares mediante cuentos maravillosos, mitos eponímicos que explican el origen de la tribu derivado del nombre de un imaginario antepasado" (Taylor, 1871, pp. 35-36). En este sentido, podemos 
decir que los textiles, reconocidos como ese grupo de hilos que se entrelazan y combinan de forma experta y con diferentes finalidades, tienen como fundamento la comprensión de "categorías sensibles que tienen que ver con la misma percepción del cuerpo, del tiempo, del espacio" (Gutiérrez, 2012, p. 22).

Así entendidos, los tejidos, y especialmente los tejidos textiles, son más que artefactos de protección, son considerados como objetos identificadores y su importancia radica en los saberes que se encuentran plasmados en ellos. Un ejemplo de ello es el vestido, uno de los principales productos del tejido; la ropa tiene como objetivo diferenciar grupos dentro de una sociedad, a veces por su pertenencia a una cultura, en otras ocasiones para distinguir la actividad de un individuo en la vida o para denotar un nivel económico o simbólico:

Los textiles indígenas mexicanos son elementos culturales que han acompañado a los pueblos originarios en un largo proceso civilizatorio, las estructuras de las telas consignan la historia y la tradición de muchas generaciones; a través del vestido y uso de los textiles se ha mostrado el poder, la estratificación social, la moda, la apreciación estética y la economía (Gómez, 2014, p.18).

La indumentaria es un lenguaje que forma parte de un sistema social; en él se encuentra un valor histórico propio de cada cultura a la que pertenece. El vestido corresponde a un identificador, es una estructura normativa en constante transformación. Este elemento puede modificarse en conjunto o de forma individual y la producción de estructuras diferentes dependerá tanto de la evolución de la sociedad como de los cambios en la técnica, los materiales y los procesos de producción.

Sin duda la indumentaria es uno de los principales símbolos de identificación entre las culturas, esto puede verse también en los pueblos del norte de México. En específico, los tarahumaras, quienes tienen particularidades que caracterizan su vestimenta entre otras etnias. Ellos se autodenominan protectores de la naturaleza y esto es un principio para sus creaciones, la explotación 
respetosa de los recursos naturales que les rodean se deja ver en la austeridad de sus prendas.

Al igual que muchos de los pueblos originarios, los rarámuri han pasado por una historia de encuentros con los conquistadores; son un pueblo que se encuentra en movimiento, pero a su vez reservado en la Sierra Tarahumara, donde practican en la privacidad de las barrancas su vida cotidiana, ritos y costumbres.

Los primeros contactos con las tribus que habitan la Sierra Tarahumara indican que andaban desnudos y utilizaban fibras semiblandas para protegerse del clima. En la etnografía de Carl Lumholtz, ${ }^{3}$ El México desconocido, se describe la indumentaria de los varones con un calzón tejido burdamente de lana, atado a la cintura con una faja de vistosos dibujos. Algunas veces complementan este vestuario con una especie de poncho pequeño de los mismos materiales y, dependiendo del clima, en invierno utilizan la cobija, objeto tejido comúnmente por las mujeres de la comunidad. Las mujeres utilizan faldas largas igualmente sujetadas por la faja, agregando una blusa tejida de lana. Tanto varones como mujeres utilizan cintas para sujetarse el cabello y huaraches manufacturados con vaqueta (Lumholtz, 2012, p. 161).

La vestimenta de los rarámuri, al igual que la de otras culturas mesoamericanas, sufre un gran cambio en el periodo de la conquista con la llegada de nuevos materiales y procesos semiindustriales. Esta indumentaria, a su vez, fue cambiando conforme evolucionó la sociedad mestiza ${ }^{4}$ y se introdujeron procesos industriales. En la colección etnográfica del Instituto Nacional de Antropología e Historia (INAH, 1992) se explica que los rarámuri con el tiempo han ido dejando de manufacturar sus propios objetos tejidos y su uso en rituales (p. 13).

3 Carl Sophus Lumholtz, teólogo y antropólogo noruego, patrocinado por el American Museum of History para el estudio etnográfico del noreste de México de 1890-1910, experto en la cultura tarahumara.

4 El término mestizaje "usualmente refiere al proceso de mezcla racial entre españoles e indígenas y a la génesis de un tipo racial y étnico particular: el mestizo... un proceso de mezcla interracial y/o intercultural, es un fenómeno que encuentra un espacio fundacional en las Américas, especialmente en esas áreas colonizadas por los españoles y los portugueses" (Quirós y Díaz, 2007, pp. 16-17). 
Actualmente, muchos varones visten pantalón de mezclilla y camisa como los mestizos; en algunas ocasiones portan su vestimenta tradicional para algunos rituales, pero los materiales han cambiado: el calzón es complementado con una blusa con corte colonial español de olanes, ambos de manta (algodón); las mujeres ahora visten una falda en múltiples capas y una blusa como la anteriormente descrita.

En ambos géneros existen elementos que los distinguen y se siguen utilizando, por ejemplo la faja, que puede ser de lana o estambre con colores más vistosos, los huaraches, ahora con un soporte de reciclado de llanta, y una cinta para recoger su cabello. Otro elemento común son las cobijas para protegerse del frío extremo, aunque en algunos asentamientos ya no se utilizan los materiales tradicionales y solo las compran en comercios mestizos.
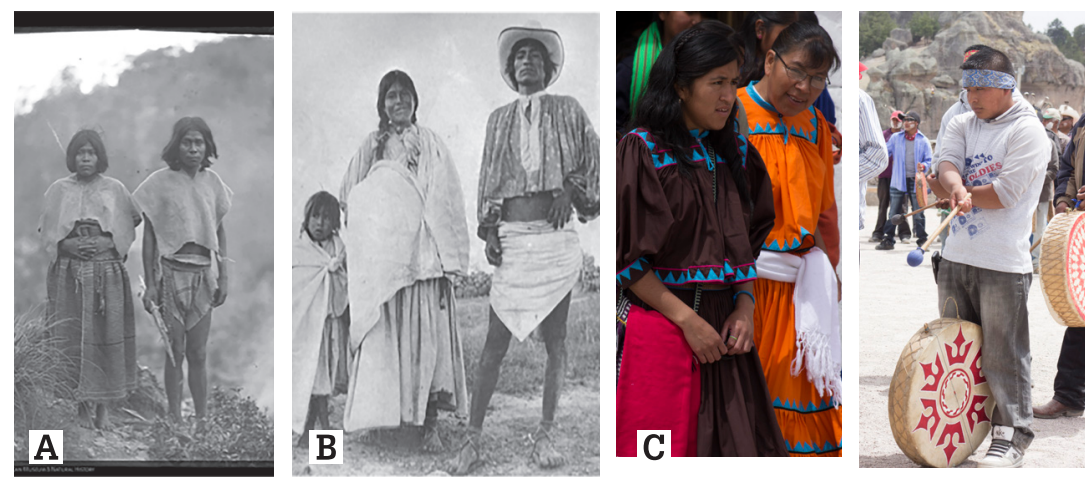

Imagen 1. A) Colección de fotografías Expedicion de Lumholtz a México 1890-1898. Museo de Historia Natural Americana. B) Los Tarahumares, Colección Etnográfica, INAH. Foto: Sandra Gutiérrez. C) Semana Santa rarámuri, Arareco, Chihuahua 2015. Fotos: Rocío Marceleño.

Desde la perspectiva de la indumentaria, los textiles emblemáticos con menos cambios en el tiempo, como la faja y la cobija, comparten un valor dentro de la cosmovisión de los rarámuri, donde el acto de tejer sigue siendo muy importante. La cosmovisión es uno de los elementos que forman el conjunto de creencias de 
una sociedad, es la forma en la que se interpreta el mundo. De la cosmogonía como sistema de creación del universo que permite a las sociedades permanecer y transmitir sus saberes, forman parte también las representaciones artísticas y la trascendencia de sus conocimientos en sus diferentes formas de expresión plástica, visual y verbal. Todo esto se refleja en los textiles de los rarámuri, donde la importancia del tejido se hace patente en las muchas horas que todavía buena parte de la población dedica a su elaboración.

\section{TEXTILES RARÁMURI}

Los textiles rarámuri son catalogados como elementos culturales, como ya vimos, identificadores. En ellos se plasman metáforas del universo y de la vida misma, se asignan diversos significados desde la cultura, la comunidad y el individuo que los porta. Para entender cómo se conforma el tejido, debe comprenderse que los hilos son el material fundamental para su creación; es el elemento mínimo que, al ser trabajado en conjunto, crea un producto mayor que tiene una carga simbólica importante para la cosmovisión de este pueblo. ${ }^{5}$

Al diseccionar los elementos de los objetos creados, los hilos forman las evidencias materiales de la tradición de tejer. En el caso de los rarámuri, aunque los objetos son pocos comparados con otras etnias, son materiales que nos permiten el estudio de su cultura con particular atención, son ellos los que logran unificar tanto lo tangible como lo intangible. Dentro de lo material y lo espiritual, muchas culturas metaforizan esta relación con los hilos que permite la conexión del hombre con su universo.

Por ejemplo, varios de los textiles rarámuri están tejidos en dos tonalidades naturales (se utiliza el material original de la lana de los borregos que se tienen en crianza, en negro y blanco) y cada

\footnotetext{
5 También otros pueblos de nuestro territorio, como los nahuas del mundo prehispánico, hablaban de hilos invisibles en las extremidades del cuerpo que los vinculaban con el cosmos. De hecho, varias culturas en el mundo han utilizado la imagen del hilo o la cuerda en relación con la vida; en las mitologías griega, romana y nórdica hay signos de ello (Pintado, 2010, pp. 90-91).
} 
uno de estos tonos tiene un significado en especial: el negro hace alusión al cosmos y la noche; el blanco a las estrellas y los caminos iluminados. Las cobijas, por mencionar otro ejemplo, "se asocian con nociones cosmogónicas, las cuatro borlas de sus esquinas, llamadas orejas, representan los pilares cósmicos en los puntos cardinales, mientras que las líneas representan los múltiples planos del universo" (Levi, 1998, p. 308).

En este sentido, es importante resaltar que, para los rarámuri, la dualidad es un elemento presente en su cosmovisión, es decir que no hay bien sin mal, día sin noche, amanecer sin atardecer; que hay una relación femenino/masculino, frío/caliente, arriba/ abajo en muchas de sus creaciones.

...el textil es la conjunción o síntesis del todo, porque en él encontramos tejidos ojos, espejos, flores, cruces, cerros, escaleras, espirales, es decir, las representaciones de los elementos estructurantes del universo y de los seres vivientes. Se trata de fuerzas contrarias, cuyo flujo se percibe como un ritmo, un ciclo entre la vida y la muerte, la creación y la destrucción (Aguilera, 2014, p. 152).

Incluso, varios de los relatos que hablan de su cotidianidad se basan en "si haces bien o si haces mal". Ellos se entienden a sí mismos como hijos de riablo y riosi (hijos de Dios y del Diablo) (Pintado, 2011, min. 19:25).

En este contexto, explicaremos a continuación algunos datos fundamentales de la relación de la vida con los hilos en los rarámuri y cómo se vacían estos significados en dos de sus textiles: la cobija y la faja.

\section{RIMUKÁ, HILOS Y SUEÑOS}

Los hilos, dentro de los mitos de creación, tienen un papel importante. Los rarámuri se consideran conectados con el mundo espiritual por medio de ellos. Como explican los expertos, "son hilos imaginarios... en las extremidades del cuerpo y, sobre todo, aquellos que salen de la mollera" (Pintado, 2012, p. 90) y son "los 
lazos que los unen con el mundo de las almas... estos lazos son los rimuká, ${ }^{6}$ los hilos que tejen la continuidad social del mundo perceptible y el no perceptible" (Rodríguez, 2012, p. 235), hilos que conectan a cada persona con el cielo. Los niños nacen con ellos igual que nacen con un alma y por ellos están unidos con sus parientes muertos. Además, son los que les permiten soñar. Pocos días después de haber nacido ${ }^{7}$ deben cortárselos (en un ritual llamado wikubema, momento en el que son presentados y se unen a su grupo) pues se cree que pueden enfermar o morir si no lo hacen. Es la misma razón por la cual se vuelven a llevar a cabo continuamente (de una a tres veces por año al menos) esos actos denominados curativos, donde se "cortan o queman" los hilos, y que tienen como primer objetivo "el de impedir los sueños con los que habitan” (Rodríguez, 2012, p. 236).

Es a través de los hilos que pueden soñar con sus antepasados, enfermar, definir su camino de vida, lo que los conecta espiritualmente al mundo de las almas, pero sobre todo, y a diferencia de otras culturas que jamás cortarían estos hilos, para los rarámuri significan "humanidad... vivir en la Tierra, tener un cuerpo, fuerzas vitales y cumplir las reglas dentro de una comunidad... el

6 Abel Rodríguez (2010) explica que originalmente se cree que los términos rimugá, rimugapu o rimugápuma proceden de rimu "sueño" y gapuma "trozar" o "partir en dos". Pero también con base en sus estudios identifica que si la voz rimuká es traducida al español por los rarámuri como "hilos" y el concepto debe proceder también del verbo rimuma, que significa "soñar", porque siempre se relaciona con este acto, es fácil entender cómo (en función de que uno de los varios significados de ká es "sombra" y que cuando ellos duermen dicen que son ligeros como el aire, parecen sombras) sería fácil sugerir que rimuká puede ser traducido también como "soñar sombras, o soñar con sombras” (pp. 236-237). Para los rarámuri, soñar se relaciona con la salud y la vida. Despertar contento o triste luego de haber soñado, puede indicar salud o enfermedad.

7 Tres días si es hombre y cuatro si es mujer, Ana Paula Pintado (2012) explica en función de sus estudios con los rarámuri de la barranca, que es porque la mujer tiene cuatro grandes fuerzas vitales o alewá -ella es la creadora de vida-, mientras que el hombre tiene tres; estas fuerzas se encuentran en el pecho (pp. 91 y 94). El tres y el cuatro aparecen diferenciando a hombres y mujeres en varios momentos de su vida, e incluso de su muerte; por ejemplo, al morir a una mujer se le hace la primera fiesta a los cuatro días, "porque necesitan más tiempo que los hombres para preparar su viaje... siendo la muerte el principio del viaje al cielo, se supone que los hombres, más ligeros para caminar que las mujeres, lo hacen más rápidamente que estas" (Plancarte, 1954, pp. 56 y 57). Los hombres necesitan únicamente tres días para prepararse; su necesidad de provisión y ayuda es mejor que en las mujeres.

Chinuahua Hoy, aÑo I 5 , Núm. I 5 (ENERo-Diciembre, 2017) 
hilo se relaciona con la iluminación espiritual, entendida como la búsqueda de la fortaleza física y emocional para vivir en la Tierra" (Pintado, 2010, p. 93). Por eso deben cortarlos, para que igual que una planta que se poda para que crezca, por medio de este ritual vivan fuertes y felices.

El owirúame ("el que cura" o curandero), es el encargado de llevar a cabo los rituales; está envuelto en una cobija durante todo el ritual. El vocablo kemá se utiliza para nombrar a la cobija, mismo "que se usa también para nombrar a la placenta" (Aguilera, 2005 en Pintado 2012, p. 104). Asimismo, se identifica la cobija con las palabras kemaka o kemala, "que también significa placenta y, más aún, emplean el término kemara para aludir al acto de tejer, lo cual es sugerente, pues relaciona al tejer con un símbolo de vida... la cobija-placenta remite a un ciclo de vida-muerte-vida" (Aguilera, 2011, p. 40). Esta metáfora de la cobija como útero o placenta tiene sentido en la protección. Una protege del frío (aunque los curanderos los usan en rituales incluso aunque haga calor) y la otra protege al feto en su desarrollo pero la metáfora se hace porque la placenta es un resguardo. En un ritual "no faltará que el curandero, al cantar y bailar, se cubra con una cobija... la cobija tiene un significado que va más allá de protegerse del frío... el curandero se comunica con los antepasados por medio de la danza y el canto, es decir, se transporta al origen", ya que se cree que es quien está conectado todo el tiempo con el otro mundo. Es por conducto de él que los demás rarámuri pueden hablar con los espíritus, a los que llaman antepasados, o ser curados de enfermedades y hechizos.

El que cura personifica a Onolúame (deidad-es) y es quien da las fuerzas vitales o alewá al recién nacido por medio del soplo, es quien corta el hilo y junta las articulaciones del niño (este ritual se denomina romilala); así es como el niño ya no es parte del mundo de origen sino que ahora es parte del mundo terrenal. El ritual se lleva a cabo con una vela encendida pasando por donde se encuentran las fuerzas vitales, entre el estómago y el pecho, llamado sula; si es mujer se pasa la vela por su vientre, luego por los hombros y las articulaciones hasta llegar finalmente al mochó 
gola o mollera. Se acompaña la vela con una cruz mojada de tesgüino; es con lo que simbólicamente se comienzan a juntar las articulaciones y para finalizar se realiza la curación que consiste en quemar, o kosma, algunos cabellos, con olotes quemados en el sahumador. A partir del momento de la curación y a lo largo de su vida, el owilúame cuidará de los sueños del rarámuri (Pintado, 2012, pp. 95-98).

La importancia de este ritual reside, como ya se dijo, en que, al seguir conectados con el mundo de las almas, se pueden enfermar y hay que evitar, dicen, que "caigan los rayos", porque las almas pueden extraviarse.

En este ritual nos damos cuenta de la relación de los hilos con su cosmos y cómo el portar la cobija por quien está conectado todo el tiempo con el mundo de las almas, es de importancia para esta cultura. En algunas otras investigaciones se menciona que se porta este textil en los rituales que son guiados por el owirúame, aunque no se adentran en muchos detalles. Sin duda este elemento cultural es fundamental para los rarámuri, además de ser un objeto de uso cotidiano.

\section{LA COBIJA PROTECTORA}

Las primeras etnografías hechas en la Tarahumara explican que la cobija tiene un papel muy importante en la vida del rarámuri. Lumholtz (1902) relata que se podían utilizar en ceremonias de matrimonio para cubrir a la novia y al novio con sus manos unidas, tanto como en rituales funerarios (en Levi, 1998, p. 307). Desde el primer registro sobre los difuntos envueltos en una cobija y puestos en una cueva con sus familiares muertos, hasta los últimos estudios que explican que hasta hace algunos años aún se les envolvía en cobijas que fueron suyas cuando vivieron, la cobija ha permanecido como un símbolo de protección y de acompañamiento.

Los rarámuri tienen la creencia de que al morir emprenden una nueva vida; para ellos se lleva a cabo un largo viaje caminando que finaliza en el cielo, lugar donde residen los muertos. 
Ellos creen en la importancia del caminar. Tanto en vida como en la muerte, dedican fiestas especiales para continuar su siguiente vida: "hay que hacer todo lo que podamos en esta vida, hay que hacer muchas cosas y caminar mucho" (Benito, 2016, Comunicación personal). Los relatos explican que en la muerte "llevan una vida semejante en muchos aspectos a la terrena, pero libre de penas y de las molestias de los chabochi”" (Plancarte, 1954, p. 56). El muerto no se va de forma inmediata, puede tardar días o meses. Hay una preparación y se celebran diferentes fiestas y ceremonias funerarias en las que se despide al difunto y se le prepara para el viaje con alimentos y protección.

Se dice también en los relatos sobre la siguiente vida, que para los rarámuri "los muertos viven del otro lado, hacia donde se pone el sol; y por eso cuando se pone el sol, acá oscurece, allá amanece" (Cardenal, 1993, p. 34). Es por eso que el cuerpo en el ritual, al ser acomodado para despedirse, así como cuando va a ser enterrado, se orienta con la cabeza hacia donde se pone el sol, el Oriente.

En el mundo de los muertos todas las cosas se realizan en forma inversa al modo en que se hacen en esta vida. Plancarte (1954) explica que la noche es día y la luna es sol, que se siembra cuando en este lado es invierno y se cosecha en otoño, es por eso que a los muertos se les dedican los alimentos con la mano izquierda, que las danzas y movimientos funerarios se realizan siguiendo la dirección contraria a la mano derecha (p. 57).

Para preparar a un rarámuri se le cierran los ojos y se cruzan sus brazos; posteriormente se envuelve en su cobija, la que usaba en vida. Al ser envuelto se coloca hacia el Oriente con cruces, para sostener el rosario y ofrecer los alimentos a su lado y el tesgüino. En su lecho esparcen ceniza, pues sus familiares y amigos quieren conocer el animal en el que se han convertido en el mundo natural, y por medio de las huellas en el polvo pueden saberlo. Una vez envuelto y amarrado en su cobija, cuando se han despedido de él, entre dos rarámuri -uno en cada extremo- lo

8 Chabochi es una palabra del rarámuri que se utiliza para referirse al hombre blanco, al extranjero. 
colocan sobre sus hombros y lo llevan a enterrar al campo santo (González, 1982, p. 126).

Sobre los mitos de creación y muerte, dentro de la cultura de los rarámuri, que involucran a la cobija, existen diversos relatos, según la región en la que se encuentran. El relato de la inundación y la cobija es mencionado por algunos cronistas, como Luis González (1987) y Jerome Levi (1998). Tomaremos un ejemplo del primero: ${ }^{9}$

Relato de la inundación y la cobija salvadora

Decían que cuando fuera a acabarse la tierra, tendrían primero que arreglar bien una cobija llamada churita. Hay dos clases de churitas: una cobija se llama ri'wítuama, está tejida del mismo modo, pero [con el hilo] más torcido. Es como una cobija llamada gawisori, pero más retorcida. Cuando la tierra pues, fuera a acabarse, cuando fuéramos a morir todos los habitantes del mundo, ya tendríamos que tener totalmente terminada la churita, porque dicen que esta es buena y esta será la que nos salve. Si nos cobijáramos con el gawisori cuando fuera a consumirse la tierra, una vez que estuviéramos así acobijados, la misma cobija nos devoraría del todo. Esto no pasaría con la churita, porque dicen que esta nos salvaría. Cuando el agua fuera llegando arriba de los cerros, que entonces nos estemos en cuevitas cobijados, tapando donde haya agujeros para que no se meta el agua. Y dicen que el agua no entrará por que la churita será nuestra salvadora (Erasmo Palma, comunicación personal en González, 1987, pp. 407-408).

El relato se relaciona con la cobija como símbolo para la vida y como elemento protector contra la muerte, pero también habla de la importancia de que sea la cobija que se usó durante la vida, no un objeto nuevo sin anclaje con las personas que mueren: "El mundo se quedaría oscuro o que el agua se iba a desparramar sobre él al final de los tiempos". Y la cobija iba a salvar al hombre de la inundación. La churita era "una cobija de segunda, [es decir, rehecha de una cobija anterior], porque esa cobija es a favor de la

9 Estos etnógrafos centraron su investigación en la zona cercana a Batopilas.

Chinuahua Hoy, aÑo I 5 , Núm. I 5 (ENERo-Diciembre, 2017) 
gente [...], porque si fuera una cobija de primera, [es decir nueva], como ellos llaman gawisori, cuando se acabara el mundo y uno se tapara con ella, esta cobija devoraría al hombre entero" (González, 1987, p. 411).

Este relato remite también a que los rarámuri son protectores del mundo natural y cuidan su entorno, por eso la importancia del uso de los recursos naturales solo para lo que es necesario en su cotidianidad: alimentación, indumentaria y protección, pues se dice que "tata Dios" podría castigarlos ahogándolos en la inundación.

\section{LOS CAMINOS DE LA VIDA, LA FAJA}

Para el rarámuri, caminar es parte fundamental de la vida cotidiana; desde pequeños, una de sus actividades es el pastoreo de chivas. Además, caminar es también su principal medio de transporte. La región serreña que lleva su nombre, la Tarahumara, tiene como características primordiales sus grandes barrancas, acantilados, cascadas y bosques. El andar es también una acción muy presente en sus creencias: "Dios fue el primero en hacer los caminos y luego ya pasaron sus hijos. Cada rarámuri tiene su camino y debe esforzarse por trabajar y hacer bien las cosas para ir por el camino de Dios, que va hacia arriba, mientras que el camino del diablo va hacia abajo" (Catarino. Comunicación personal 2004 en Aguilera, 2011b, p. 83).

El caminar, para los rarámuri, es una constante dentro de su pensamiento mágico. Por ejemplo, en el ritual de muerte mencionado anteriormente se dice que un rarámuri nunca deja de caminar en la siguiente vida. "Entre los rarámuri se explica que todo tiene un camino (el sol, la luna, las nubes, las estrellas, el agua, los seres vivos, etc.), incluso los curanderos transitan a otros planos del cosmos utilizando ciertos caminos" (Pintado, 2012, pp. 108-109). El camino es una metáfora de la vida que se vacía en la prenda rarámuri conocida como faja: 
Los diseños que conforman la faja, así como la faja misma, son representaciones cosmogónicas de gran trascendencia, de tal forma que cada uno de los elementos gráficos refiere a conceptos relacionados con el momento de la creación y de la comunicación entre los diferentes planos del cosmos asociados con la noción tarahuamra del camino de la vida (Aguilera, 2011a, p. 211).

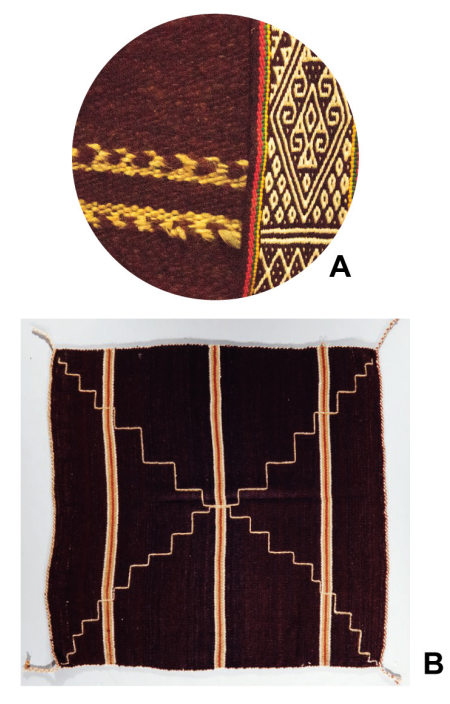

Imagen 2. A) Textiles con diseños geométricos, aludiendo al cosmos y el camino. Museo Regional de Creel. B) Cobija tarahumara. Recolectada entre 1965 y 1987 por Eugene H. Boudreau en el Municipio de Batopilas, Chihuahua, México.

Ana Paula Pintado coincide:

El proceso de la vida, como el de la elaboración de la faja, es creativo y debe seguir reglas precisas... al hacer la faja se busca el entrelazamiento y unión de los hilos [...] La imagen de la faja es el reflejo de los caminos de la vida; asimismo, el cuerpo humano tiene sus caminos, y mientras unos deben cortarse, otros deben fortalecerse o juntarse tal como se haría con una faja (Pintado, 2012, p. 105). 
La faja es parte de la indumentaria de muchos pueblos indígenas de México, "es el complemento imprescindible del enredo ${ }^{10}$ pues permite sujetarlo a la cintura. Independientemente de su propósito práctico, algunos grupos han investido a la faja de poderes mágicos... existe una sensación imprecisa de que la prenda brinda protección a su portadora" (Lechuga, 1997, p. 156). Es muy común su uso en las mujeres, pues es complemento de la falda, pero en los rarámuri esto no puede generalizarse, porque la faja la utilizan las mujeres solo en algunas regiones. ${ }^{11}$ Quienes la utilizan principalmente son los hombres y los curanderos, también en la cintura.

La palabra que se utiliza para faja en rarámuri es puri o puraka, que se deriva de pura, "atar", y alude al hecho de que este objeto se ata a la cintura. Sin embargo, su apreciación entre los tarahumaras va mucho más allá del uso práctico. El trabajo La faja ralámuli, un entramado cosmológico, de Sabina Aguilera (2011b) permite entender la función y significado de este objeto a través de la descripción de su composición plástica, su organización y combinaciones. La autora logra develar parte del simbolismo detrás de la faja a partir de categorías mitológicas y rituales. De los datos más interesantes, nos permitimos rescatar el que tiene que ver con el ciclo de la vida:

La constante en los diseños y composiciones es la noción de movimiento, es decir, lo que genera vida o lo que permite su permanencia... ese movimiento pertenece a un ciclo de vida-muerte-vida, que afecta a los hombres, a los animales, a las plantas, a los astros y a la existencia en general. Es lo que mantiene un balance o un equilibrio cósmico (Aguilera, 2011b, p. 131).

Sobre la relación del tejido con el cosmos, la autora explica también que los hilos que conforman el universo se entienden como interconexiones que permiten el movimiento de los astros

10 El enredo es una falda de origen prehispánico que consiste en un rectángulo de tela, a veces abierto, otras cosido en forma de tubo (Lechuga, 1997, p. 156)

11 Se dice que la carga simbólica de la faja es muy fuerte para una mujer y no cualquiera puede con ello (José Luis Flores. Comunicación personal . Creel, Chihuahua, 2016). 
"como los caminos a través de los cuales las deidades, almas y demás entidades pueden desplazarse en las diferentes dimensiones" (Aguilera, 2014, p. 108). Su trabajo permite entender la iconografía, entre la que la que el cerro como matriz o contenedor de agua, la escalera como camino, las figuras romboides como centro (eje o portal) y el camino en espiral, propio del pensamiento mágico del rarámuri, son las formas primordiales que hablan del orden del universo.
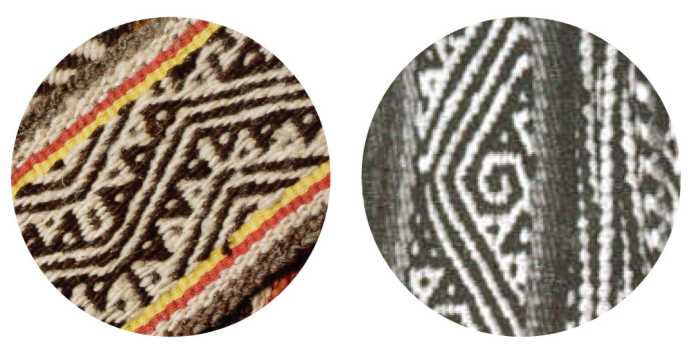

Imagen 3. Fajas con representaciones de cerros y caminos. Foto 1: Artes de México Tarahumaras. Foto 2: Los Tarahumares, Colección Etnográfica, INAH.

Se concluye que, a través de este otro objeto, tejido también en el contexto de la relación de los hilos con la vida, se representa una forma de ser y de ver el mundo; el proceso de creación de la faja rarámuri "implica necesariamente un conocimiento cosmogónico y tecnológico que, al ser plasmados en el objeto creado, reproducen a la cultura en su conjunto" (Aguilera, 2011b, p. 61). Es en este sentido un objeto de uso práctico y estético con un vasto contenido simbólico. 


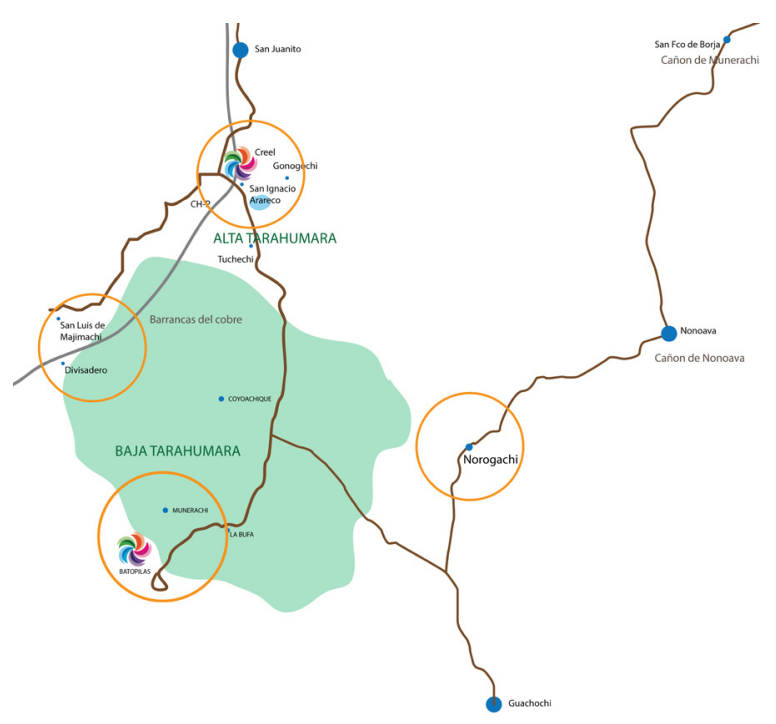

Ilustración 1 Mapa de las zonas de producción textil orientada al turismo. Chihuahua, abril 2016. Rocío Marceleño.

\section{TEJEDORAS}

En 2016 se realizaron una serie de visitas de campo en la alta y baja Tarahumara, en las comunidades cercanas a los pueblos mágicos Creel y Batopilas, para tener un acercamiento con artesanas tejedoras de esa región. La mujer es quien principalmente realiza esta actividad artesanal ${ }^{12}$ y es en esta zona donde hay

12 Es común que la actividad de tejer se relacione con el mundo femenino. En México, antes de la colonia, "todas las mujeres, ricas o pobres, tenían la obligación de hilar y de tejer" (Stresser-Péan, 2012, pp. 231). Pues es en ella donde recae este saber-hacer en las prácticas cotidianas. En México, en la región de Mesoamérica, los códices brindan evidencia arqueológica de las deidades que representan el tejido; los aztecas tenían una patrona especial para los trabajos textiles a la que llamaban Xochiquétzal, la primera mujer que había hilado y tejido, la cual se representa sentada frente a un telar (Johnson, 2005, p. 8), quien era la diosa de la fertilidad y la vegetación. Por otra parte, en el códice Borgia, Tlazoltéotl era la diosa mexica del tejido y era representada con uno o dos malacates que tenía a manera de adorno en su peinado. Además, era la diosa del parto y la lujuria (Stresser-Péan, 2012, p. 232). Para los mayas la diosa Ixchel, diosa de la luna y esposa del sol, patrono del hilado, era también conocida como "la 
una importante producción, especialmente de artes turísticas. ${ }^{13}$ El objetivo era tener un acercamiento práctico para entender su quehacer y las diferentes visiones que tienen ellas mismas de su trabajo creativo.

Aguilera (2011) explica que en el tejido rarámuri,

Tanto el proceso creativo como el objeto terminado tienen mucho que decirnos. El solo hecho de que los tarahumaras continúen utilizando el telar y tejiendo diseños que datan de tiempos prehispánicos y que están cargados de un importante y fuerte simbolismo indica que debe hacer algo más que el simple gusto por tejer ciertos diseños en determinados textiles. En definitiva, es arte y conocimiento, no solo es uso técnico y repetitivo (p. 61).

En nuestro acercamiento pudimos darnos cuenta que en la práctica del tejer existen tradiciones familiares, sociales y étnicas, que permiten a la tejedora conectar el cuerpo con la esencia del ser (como individuo y como miembro de un grupo), pero hay además, en las tejedoras rarámuri que conocimos, un claro gusto por el tejido.

Las mujeres han tenido un lugar preponderante en el tejido. La actividad de tejer no es exclusiva de ellas, pero ha sido considerada por muchas culturas como una de las tareas habituales para este miembro de la familia que tradicionalmente ha ocupado el lugar de cuidadora de los hijos y de la casa. La mujer forma parte esencial de las sociedades, aunque directamente no se les da el crédito de ser las generadoras de la identidad de muchos tejidos, es muy claro que los motivos que plasman en sus telas tienen que ver con sus actividades diarias y con su propio pensamiento (Ramírez, 2012, p. 39). Esta actividad de saber-hacer es de vital

de las trece madejas de tela a colores" (Johnson, 2015, p. 8).

13 Se participó también con la asociación civil Centro de Desarrollo Alternativo Indígena, Cedain. Ellos trabajan directamente con artesanos dentro de la Sierra Tarahumara. Con su colaboración, en un primer acercamiento se contactaron artesanos con los que realizan actividades importantes y de sus registros pudimos seleccionar y contactar a mujeres tejedoras que trabajan diferentes materiales y que tienen producción artesanal constante, ubicadas dentro y cerca de los puntos turísticos de mayor interés.

Chinuahua Hoy, año I 5 , Núm. I 5 (ENERo-Diciembre, 2017) 
importancia, ya que el conocimiento de la técnica se transmite de generación en generación y se le enseña desde muy temprana edad a las niñas, junto con las labores del hogar (Chamoux, 1992, p. 26). En el caso de nuestras informantes esto es común, sus madres les enseñaron a ellas y las que ya tienen hijas o hermanas pequeñas han hecho lo mismo.

Concimos ocho tejedoras en total. Con algunas se dio la oportunidad de visitar sus casas y conocer a sus familias; con la mayoría se pudo realizar una charla sobre su tejido y registrar su actividad mientras la realizaban. Las entrevistas arrojan información sobre su forma de vida y su trabajo textil. Aunque no es posible vaciar aquí cada dato y la riqueza de cada experiencia, de lo más relevante relacionado con los aspectos antes vistos en este texto, podemos rescatar una distinción importante entre las comunidades que se encuentran más alejadas de las ciudades de los chabochi y las que encuentran cerca de las ciudades. La recolección de materiales y la producción artesanal se torna más difícil estando lejos; por ejemplo, las tejedoras de ware (cestería) para conseguir sus materiales, recorren largas distancias fuera de sus hogares, ya que son muy escasos. Como esta actividad se realiza para la venta al turismo, cada vez se vuelve más difícil encontrar el material de manera silvestre. Por el contrario, es más fácil conseguir material industrializado cerca de las ciudades. Con ellos trabajan incluso otro tipo de tejidos que no son los que tradicionalmente fabrican para autoconsumo; esto les ha permitido tener variedad de productos para el turismo, que es su principal comprador.

\begin{tabular}{c|c|c|c|c|c|c|c|c}
\hline \multicolumn{2}{l}{ Artesana } & Comunidad & Tejido & \\
\hline \multicolumn{2}{l|}{} & Cestería & Morral & Pulsera & Faja & Cobija & Bordado & Chaquira \\
\hline Agripina & Gonogochi & & $\mathrm{x}$ & $\mathrm{x}$ & $\mathrm{x}$ & & $\mathrm{x}$ & \\
\hline Lucía & Tucheachi & & $\mathrm{x}$ & $\mathrm{x}$ & $\mathrm{x}$ & & & $\mathrm{x}$ \\
\hline Luisa & Arareco & & $\mathrm{x}$ & $\mathrm{x}$ & $\mathrm{x}$ & $\mathrm{x}$ & & \\
\hline Carmen & Munerachi & $\mathrm{x}$ & & & & & & \\
\hline Catalina & $\begin{array}{c}\text { San Luis } \\
\text { Majimachi }\end{array}$ & $\mathrm{x}$ & & & & & & \\
\hline
\end{tabular}




\begin{tabular}{c|c|c|c|c|c|c|c|c}
\hline Cande & Tucheachi & & $\mathrm{x}$ & $\mathrm{x}$ & $\mathrm{x}$ & $\mathrm{x}$ & & \\
\hline Sabina & Creel & & $\mathrm{x}$ & $\mathrm{x}$ & $\mathrm{x}$ & $\mathrm{x}$ & $\mathrm{x}$ & \\
\hline Rosa & Panteón & & $\mathrm{x}$ & $\mathrm{x}$ & $\mathrm{x}$ & $\mathrm{x}$ & $\mathrm{x}$ & \\
\hline
\end{tabular}

Algunas de nuestras informantes trabajan de manera formal la artesanía; otras hacen sus productos cerca de casa y luego venden sus productos en las zonas turísiticas. Lo más común de cualquier forma es que tejan al aire libre. Sus materiales y telares varían según los tipos de tejido que realicen. Por ejemplo Cande y Luisa, quienes junto con sus familias se dedican al tejido y explican sobre los telares: "Si es un morral agarramos uno mediano y lo recargamos a la pared, si son fajas y pulseras tomamos el chico y tejemos afuera y hacemos varias piezas, el de troncos solo para hacer cobijas, pero casi no nos la compran por que salen muy caras de hacer" (Cande. Comunicación personal, abril 2015). Luisa es quien se encarga de vender los productos en el lago de Arareco, pues es un punto muy turístico cerca de algunas tiendas de artesanía. "A veces se paran camiones con los turistas y nos piden mucho trabajo para llevar, siempre tenemos que tener fajas y pulseras listas" (Luisa. Comunicación personal, abril 2015).

En este sentido vale la pena mencionar dos datos importantes sobre las mujeres que conocimos: su ejercicio es fundamental para lograr su sustento y para ellas queda muy claro el papel que desempeñan en su familia. Agripina, por ejemplo, tiene 18 años, es tejedora de faja en un telar de marco, es la mayor de seis hermanos y ayuda a mantener su casa. Además de producir artesanía, trabaja como tendera en la caseta de Cedain de su comunidad. Ella se encuentra en constante capacitación acerca de cómo hacer y vender su artesanía para ofrecerla al turismo a un precio competitivo. En su discurso de venta refleja el valor del trabajo en la elaboración de estos objetos, por ejemplo, resalta el tiempo de elaboración de cada pieza, para que la gente se dé cuenta de la complejidad del proceso. Su madre está con ella gran parte del tiempo, aunque no habla castellano. Agripina explica que en su cultura la mujer debe de seguir sus caminos como esposa y aten- 
der su casa, su marido y sus hijos; ella al final del día se toma un tiempo para trabajar la artesanía en conjunto con miembros de su familia, a quienes les enseña.

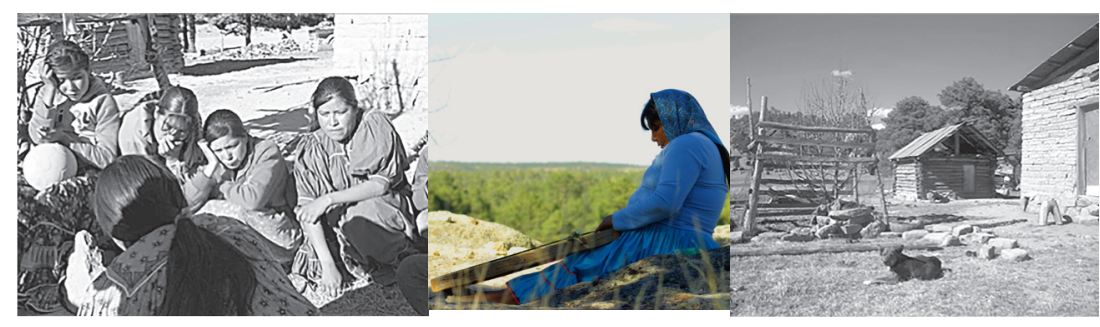

Fotografía 2 Lucía tejiendo desde su lugar de trabajo. Bocoyna, abril 2016. Documenta.

Laura, quien es también artesana, pero de frutos envasados, comenta sobre el camino de una mujer: "primero atiendo a mi marido, a mis hijos y a mi casa, cuando alcanza el tiempo hago artesanía" (2016). La historia de Lucía no es tan diferente de las mujeres de la región. Su papel primordial es servir a su marido, pues sus hijos se encuentran en los internados en las diferentes zonas de la alta tarahumara. Ella comenta que su marido le hizo el marco [bastidor de tejido], para que le ayudara con la casa. Después de un tiempo vio que lo hacía bien y él dejó de trabajar, una acción no poco común de otros hombres de la región. Las mujeres han tomado un papel fundamental en el sustento de sus hogares y tejer se ha convertido en una actividad importante para proveer.

Son mujeres amas de casa y proveedoras del sustento; tejiendo vacían su bagaje cultural y personal. Es importante entender que tienen un papel como creadoras y el tejido es una actividad donde también se han refugiado y expresado de manera silenciosa; esto les permite una exploración personal y un ejercicio importante de relación con el entorno, desde conseguir su material hasta convertir su resultado en un producto con valor estético importante; productos que vacían de alguna forma el placer de crear, de hacer un tejido de ellas mismas. Sus pensamientos y preferencias se vierten en sus decisiones sobre el color, las formas y las composiciones. 
Así es como logran construir un espacio propio de creación que al mismo tiempo, como vimos anteriormente, conforma una declaración sobre su pueblo.

En cada tejedora se puede observar también la dedicación y fuerza de su expresión. El tejido no es un ejercicio fácil, pero su preocupación por la conservación de su tradición, por mantener vivo este saber-hacer y que las futuras generaciones no pierdan sus costumbres, les lleva a seguir su actividad con mucho empeño.

\section{A MANERA DE CONCLUSIÓN}

El tejido, a partir de las últimas tres o cuatro décadas del siglo $\mathrm{XX}$, tomó un valor diferente al ser un ingreso extra para el sustento de las familias rarámuri. La participación de la mujer (principalmente) en las artes turísticas, ha sido observada en diferentes estudios desde entonces, así como la evolución de estos tejidos en su forma, representaciones y usos.

\section{TEJER PARA LOS RARÁMURI}

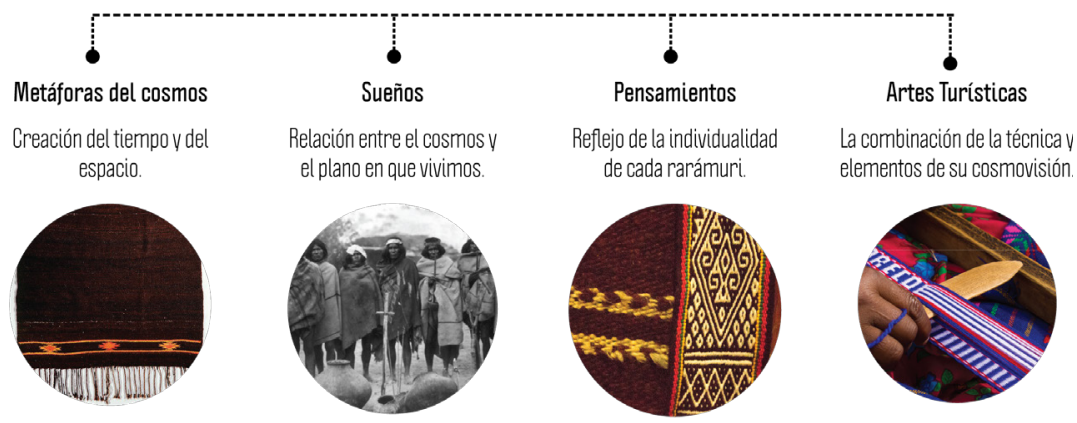

Esquema 1. Tejer para los rarámuri. Elaborado por Rocío Marceleño

Tejer, para los rarámuri, es una actividad cotidiana y llena de significado. La importancia del tejido se ve en la transferencia de saberes, los motivos que plasman y el uso de los objetos, tanto los que ellos utilizan (en la cotidianidad o en ritos) como los que son para otros, por ejemplo los turistas. Las formas y los cambios 
de materiales en la actualidad permiten observar estos objetos no solo desde la antropología como disciplina per se del estudio de estos saberes y su preservación, sino también desde el arte y el diseño, donde se ha tenido una relación importante que se enmarca en un ejercicio que promueve, optimiza y difunde los objetos creados para que su producción continúe y sea valorada.

Así pues, dar a conocer estas técnicas textiles resulta importante para su reconocimiento y permanencia, porque además de que observar los materiales y herramientas que utilizan los rarámuri permite conocer su entorno, saber qué y cómo tejen nos habla de su cultura, de la trascendencia de este saber-hacer.

Desde la perspectiva de las artes la representación de elementos, en este caso en los motivos tejidos, es un embudo de saberes que han sobrevivido al paso de las generaciones; la transferencia de la técnica se suma al simbolismo del ritual; las vivencias actuales, tanto de la etnia como de los creadores tejedores, se plasman a través de los textiles y la gráfica como elementos culturales de valor.

Como vimos anteriormente, el acto de tejer en los rarámuri va más allá una actividad técnica para sustentar la necesidad de cubrirse. Se trata de un evento de creación de objetos que reflejan su cosmovisión y la forma en que representan su propia vida; desde su nacimiento, en el que al rarámuri deben cortársele los hilos de su cabeza que lo conectan al cielo para tener una vida terrenal plena, hasta su muerte donde se prepara para otra vida, la importancia del hilo y los tejidos está presente.

Como explica Sabina Aguilera (2012), hilar para los rarámuri implica todo un proceso creativo para dar forma a la materia, que requiere una importante organización cognitiva y la experiencia para expresar su cosmovisión de manera significativa:

Así, se entiende por qué una actividad como el hilar o el tejer es una donde la memoria colectiva opera como proceso que no está fijo en el pasado, sino que, al integrar las experiencias, se renueva, reinterpreta y reproduce. Finalmente, desempeñar estas y muchas otras acciones sigue y revive aquel camino establecido en la época 
primordial, pero al ejecutarlas se vuelven únicas en tanto posibilitan el aprehender, comprender y dar significado al mundo vivido (Aguilera, 2012, p. 83).

En las representaciones de los rarámuri hay una constante relación entre vida y muerte, con los hilos y tejidos resguardan estas representaciones, que llamaremos artísticas, como lo sugiere Olmos (2005) en sus análisis de la estética de las culturas del noroeste. El autor describe que así como el arte tiene fundamentos míticos, las manifestaciones y representaciones en las sociedades indígenas también. La diferencia es que en las útimas esto tiene un impacto en el comportamiento del grupo étnico, ya que lo regulan de forma muy clara:

Las manifestaciones artísticas indígenas... poseen algunas cualidades que pudieran resumirse de la siguiente manera: a) mantienen relación intrínseca entre el arte y el sistema de creencias; b) el arte es de carácter simbólico y refleja elementos míticos vigentes en el pensamiento indígena contemporáneo y c) el código de comunicación es ampliamente conocido y socializado entre los miembros de la comunidad (Lévi-Strauss, 1970 en Olmos, 2005, pp. 51 y 52).

Así pues, estos y otros estudios existentes muestran que hay una articulación de los saberes, códigos de creencias y de conducta con todo un sistema de representación estética. "El mito, en tanto fenómeno colectivo, articula simbolismos ligados con los afectos y con la creación artística... el principio que caracteriza al arte indígena es la relación entre la manifestación sensible y el mito como fundamento del sistema de creencias" (Olmos, 2005, p. 54). En los hilos, tejidos y objetos (como la cobija y la faja), de los rarámuri podemos ver claramente ese pensar colectivo y cómo se manifiesta de forma física y simbólica en sus creaciones, principalmente en la indumentaria que utilizan para sus ritos.

El perfil del creador, en este caso la tejedora rarámuri y su contexto de vida, familia, acercamiento religioso y cotidianidad, le permiten tener un cúmulo de saberes y experiencias que proveen 
los medios suficientes para la representación y el cumplimiento de actividades primordiales en su vida. Sus objetos tejidos y los elementos iconográficos que vacían en ellos hablan de su existencia individual y colectiva y al tiempo los acercan a un mundo no material. Por todo ello, el estudio de los tejidos en la cultura de los rarámuri nos aproxima a la riqueza de una cultura, pero también a la magia de la creación como una actividad humana imprescindible para nuestra existencia.

Los productos textiles que se han analizado en esta investigación plasman una visión particular de creación propia de la identidad rarámuri. Este estudio ha avanzado al valorizar los conocimientos trabajados y valorando a los artesanos y su vivir creativo, pues son ellos quienes se encuentran dispuestos a seguir luchando en la preservacion, defensa y transformación su cultura tomando sus objetos artesanales, en particular los textiles, cuyo valor hemos resaltado en la representación de su pensamiento mágico y que los identifica abriéndose paso por el mundo.

\section{REFERENCIAS}

Aguilera, S. (2014). Textiles ralámuli. Hilos, caminos y el tejido de la vida. Berlín: Gebr. Mann Verlag.

(2012). Tejiendo conocimiento y recreando al mundo. Un análisis comparativo de los textiles tarahumara. En Gutiérrez del Ángel (ed.) Hilando al norte. Nudos, redes, vestidos, textiles (pp. 47-88). México: El Colegio de San Luis/El Colegio de la Frontera Norte.

-_ (2011a). De caminos, espejos y fajas. Un análisis comparativo de la iconografía textil tarahumara. En Bonfiglioli, C., Gutiérrez, A., Hers, A. y Olavarría, M. (coords.) (2011). Las vías del noreste III: Genealogías, transversalidades y convergencias (pp. 207-232) México: Instituto de Investigaciones Antropológicas, UNAM.

-_ (2011b). La faja ralámuli: Un entramado cosmológico. México: 
Instituto de Investigaciones Estéticas, Instituto de Investigaciones Antropológicas, UNAM.

Cardenal, F. (1993). Remedios y prácticas curativas en la Sierra Tarahumara. México: Editorial Camino.

Chamoux, M-N. (1992). Trabajo, técnicas y aprendizaje en el México indígena. México: Centro de Mexicanos y Centro Americanos, Centro de Investigaciones y Estudios Superiores en Antropología Social.

Gómez, A. (2014). La producción textil indígena entre los nahuas de Hueyapan, Puebla. En González (coord). Cambio y continuidad en las organizaciones indígenas textiles femeninas. Del capital social a la tradición textil (pp. 15-36). México: Conaculta.

González, L. (1982), Tarahumara. La sierra y el hombre. México: SEP/ Fondo de Cultura Económica.

González, L. (1987). Crónicas de la Sierra Tarahumara. México: SEP.

Gutiérrez, Donaciano y Gutiérrez, Josefina (1992). Los tarahumares. Catálogo de las colecciones etnográficas del Museo Nacional de Antropología. México: Instituto Nacional de Antropología e Historia.

Gutiérrez del Ángel, A. (ed.) (2012). Hilando al norte. Nudos, redes, vestidos, textiles. México: El Colegio de San Luis/El Colegio de la Frontera Norte.

Jhonson, K. (2015). Saberes entrelazados. La obra de Irmgard Weitlaner Johnson. Artes de México. México: Conaculta.

Lechuga, R. (1997). El traje indígena de México. México: Panorama Editorial.

Levi, J. (1988). The bow and the blanket: religion, identity and resistance in Rarámuri material culture. Journal of Anthropological Research, Vol. 54, No. 3 (Autumn, 1998), pp. 299-324. University of New Mexico. Recuperado de: http://www.jstor. org/stable/3630650

Lumholtz, C. (2012). El México desconocido. Tomo I. Volumen 9 , México: Comisión Nacional para el Desarrollo de los Pueblos Indígenas.

Olmos, M. (2005). El viejo, el venado y el coyote. Estética y cos- 
mogonía: hacia una arquetipología de los mitos de creación y del origen de las artes en el noreste de México. México: Colef/ Fondo Regional para la Cultura y las Artes del Noreste.

Pintado, A (2012). El tejido de la vida: rimuwaka y la creación de la humanidad. En Gutiérrez del Ángel (ed.) Hilando al norte. Nudos, redes, vestidos, textiles pp 89-108. México: El Colegio de San Luis/El Colegio de la Frontera Norte.

Pintado, A. (2011). Entre riosi y riablo, un documental sobre la Tarahumara. México: Meta Films.

Plancarte, F. (1954). El problema indígena tarahumara. México: Ediciones del Instituto Nacional Indigenista.

Quirós, R. y Díaz, D. Mestizaje, indígenas e identidad nacional en Centroamérica: De la Colonia a las Repúblicas Liberales. Cuaderno de Ciencias Sociales, 143. Costa Rica: Facultad Latinoamericana de Ciencias Sociales (Flacso).

Ramírez, A. (2014). Tejiendo la identidad. El rebozo entre las mujeres purépechas de Michoacán. México: Conaculta.

Rodríguez, A (2012). Los rimuká, "hilos de vida y muerte" elemento cardinal en la etiología rarámuri. En Gutiérrez del Ángel (ed.) Hilando al norte. Nudos, redes, vestidos, textiles, pp. 235-256. México: El Colegio de San Luis/El Colegio de la Frontera Norte. Stresser-Péan, C. (2012). De la vestimenta y los hombres. Una perspectiva histórica de la indumentaria indígena en México (trad. de Ángela Silva y Haydée Silva), México: Fondo de Cultura Económica, CEMCA, Museo Textil de Oaxaca.

Taylor, E. (1871). La ciencia de la Cultura. En Kahn, J. S. (ed.). (1975). El concepto de cultura (pp. 29-46). Barcelona: Anagrama.

Velasco, G. (1995). Origen del textil en Mesoamérica. México: Instituto Politécnico Nacional. 